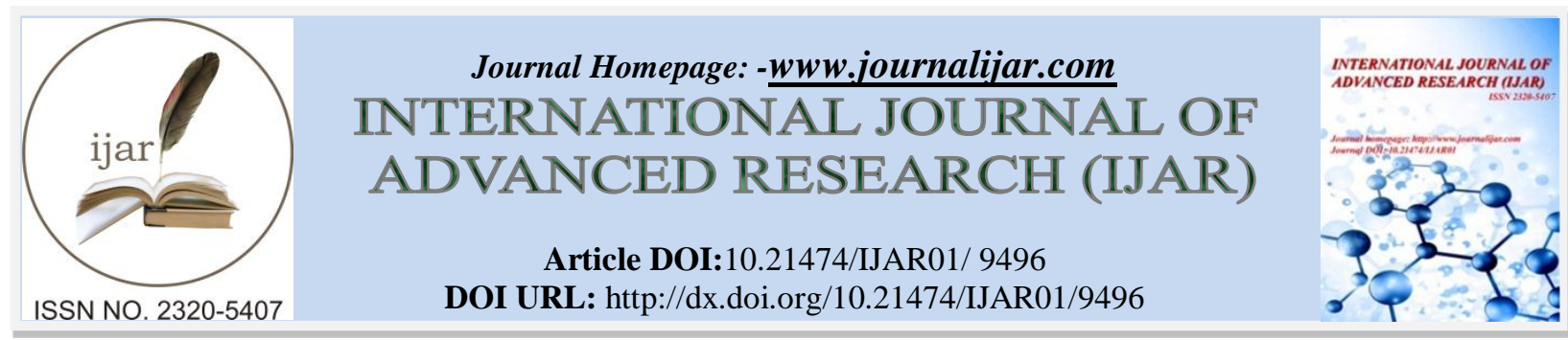

RESEARCH ARTICLE

\title{
MANAGEMENT OF ANTIDEPRESSANT INDUCED SIDE EFFECTS.
}

\section{Bhavya K Bairy ${ }^{1}$ and Laxminarayana K Bairy ${ }^{2}$.}

1. Assistant Professor, Department of Psychiatry, National Institute of Mental Health and Neurosciences, Bangalore-560029, Karnataka, India.

2. Associate dean and Chairperson Pharmacology, RAK College of Medical Sciences, RAK Medical and Health Sciences, Ras Khaimah, P.O.Box 11172. United Arab Emirates.

\section{Manuscript Info}

\section{Manuscript History}

Received: 05 June 2019

Final Accepted: 07 July 2019

Published: August 2019

Key words:-

antidepressants, side effects, depression.

\section{Abstract}

Over the last two decades, the safety and acceptability of antidepressants have been enhanced considerably. However, the adverse effects due to antidepressants are still challenging. Majority of the antidepressants cause annoying side effects when prescribed to patients with depression. These side effects often form hurdles to achieving remission of depressive episodes. Quite often the frequency of side effects are underestimated and nearly $25 \%$ of patients discontinue their treatment because of hard-to-accept adverse effects; others may continue on medication but experience reduced quality of life due to worrying adverse effects.

Proper controlling of adverse effects improves adherence, warrants suitable dosing, increases patient well-being and stops early termination of therapy. Suitable management comprises thoughtful drug assortment, expectation of common and rare but serious adverse effects and attempting for the lowest effective dose and simplest drug schedules reliable with appropriately robust management.

Copy Right, IJAR, 2019,. All rights reserved.

\section{Introduction:-}

Globally about 300 million suffer from depression (WHO, 2017). Antidepressants are a group of drugs that are prescribed most frequently. About 6-10\% of the world's population are treated with antidepressants, more so in developed countries (Wouters et al, 2014). An important consideration in the choice of an antidepressant is its safety and tolerability (Wang et al, 2018). Available data suggests that most of the antidepressants may produce troublesome adverse effects in a significant number of patients, resulting in non-compliance among those who receive these drugs. Treatment failure rates are high, projected to be between thirty-one percent and sixty percent, and are possibly controlled by a balance between alleged benefits and disadvantages (Wouters et al, 2014, Demyttenaere et al 2001). In spite of this, the adverse effects are not recognised by the clinicians. At least one adverse effect is seen in more than eighty percent of patients and majority of patients experience four different adverse effects. These adverse effects pose considerable glitches and badly disturb patients' daily routines (Hu et al, 2004). Further, it is reported that in almost twenty percent of the cases, there is doubt regarding the efficacy of antidepressants when compared to the adverse effects produced by them (Wouters et al, 2014). 
Recently introduced antidepressants have shown potential of better adverse-effect profile in comparison to the older Tricyclic antidepressants (TCAs). But of late, there is some doubt regarding this. The problem of adverse effects might have altered, but it did not reduce. The most commonly occurring adverse effects, in patients using different types of antidepressants, in descending order are loss of libido, dry mouth, sweating, forgetfulness, drowsiness, diminished concentration, restlessness, insomnia, gastro-intestinal complaints, erectile dysfunction, delayed ejaculation, dizziness and weight gain.

As adverse effects has great influence on patient compliance, response to treatment and quality of life, a thorough knowledge of management of adverse effects is necessary for best treatment of depressed patients.

\section{Jitteriness syndrome}

The jitteriness syndrome (shakiness, increased anxiety, irritability and insomnia) can develop with low doses of TCAs in patients who are sensitive to these drugs. It may happen immediately in up to sixty five percent of patients put on serotonergic or noradrenergic antidepressants (Sinclair et al 2009). Patients may discontinue antidepressant treatment if they become convinced that jitteriness will worsen their condition. Starting with a low dose and titrating slowly, mainly in patients with anxiety symptoms, might avoid the jitteriness. Alternatively, this can be managed temporarily by concomitant treatment with benzodiazepine or propranolol or waiting for tolerance to develop as jitteriness is time-limited.

\section{Gastrointestinal problems}

About $25 \%$ of patients may complain of nausea soon after starting the treatment with newer antidepressants. Patients on venlafaxine and selective serotonin reuptake inhibitors (SSRIs) are more likely to have gastrointestinal problems than with bupropion, mirtazapine, or reboxetine (Kelly et al 2008). This is not a problem as nausea disappears after two or three weeks, but it continues longer in about $30 \%$ of patients. Nausea can be minimised by taking these medications in divided doses, taking them on full stomach or taking more of the dose before going to bed. Nausea may be reduced by taking small quantity of ginger while taking ranitidine or omeprazole (Kelly et al 2008). Adding low-dose mirtazapine, a noradrenergic and specific serotonergic antidepressant to the treatment schedule may also help in reducing the chances of nausea (Uguz, 2013). Mirtazapine, unlike other antidepressants will not cause nausea, at the same time has better efficacy and earlier onset of action (Matreja et al 2012).

About $15 \%$ of the patients receiving antidepressants may complain of diarrhoea and may require antidiarrheal agents for the management of diarrhoea. Persistent diarrhoea in spite of treatment may necessitate a switch to another antidepressant. About $5 \%$ of patients may have problem of constipation especially those on TCAs. This can be treated by simple exercise, plenty of fluid and fibre rich food intake, or laxatives (Wouters et al 2014).

\section{Weight gain}

Weight gain is a regular side effect in patients who are taking antidepressants for long time except those who are put on bupropion. It is the only antidepressant that induces weight loss. Initially there may be transient weight loss in these patients which is followed by weight gain. Mirtazapine, amitriptyline, and paroxetine are well known for significant weight gain whereas other antidepressants cause only marginal weight gain. Nutritional counselling and physical exercise may be advised in patients who gain weight. Quite often one needs to switch the antidepressants to avoid weight gain. But in cases of better response to one drug, switch may not be a choice. Other measures to prevent weight gain such as adding bupropion or a psychostimulant may help in patients who are responding better to the antidepressants (Kelly 2008).

\section{Sweating}

Excessive sweating is a problem in almost $20 \%$ of patients taking antidepressants (Marcy and Britton 2005). It may be present during the entire treatment period and most commonly seen in the chest, neck, scalp and face and usually occurs with intermittent spurts. It is more common with reboxetine, venlafaxine, and bupropion. The pathophysiology is multiple hence drugs with different mechanism of action are tried to manage excessive sweating namely, cyproheptadine, a serotonin antagonist or clonidine an $\alpha_{2}$ agonist, benztropine and glycopyrrolate, anticholinergics can be beneficial in managing excessive sweating.

\section{Sexual dysfunction}

Another common long-term problem with antidepressants is sexual dysfunction. Patients may suffer from loss of sexual drive, arousal, orgasm and ejaculation (Zajecka, 2001). However, to a great extent these adverse effects are 
underreported, the main reason being physicians' reticence to address this topic. Montejo-Gonzalez et al observed that fifty-eight percent of patients had sexual problem when clinicians asked them directly, whereas only fourteen percent of patients reported sexual problem on their own (Montejo-Gonzalez et al 1997). In another study, about $34 \%$ of patients complained of sexual problems when asked directly through closed-ended questions and nearly half of them reported that the sexual problem was really bothering them (Hu et al 2004). By the end of the second week, $70 \%$ of patients experienced sexual dysfunction and it was almost $80 \%$ by 3 months treatment. The degree of sexual dysfunction differs among various antidepressant agents. A trial with more than 6000 subjects, bupropion and nefazodone reported to have lowest rate of sexual dysfunction when compared to other antidepressants (Clayton et al 2002). In another comparative study involving nearly 1000 patients, paroxetine reported to be having highest rate of sexual problem among SSRIs(Montejo et al 2001).

Sexual problems may will vanish naturally as the treatment is continued. If not a dose reduction may help to improve them. In about 50\% of patients stopping the drug temporarily for few days has reported to improve sexual problem.(Rothschild, 1995). Phosphodiesterase V inhibitors such as sildenafil or tadafil are playing a major role in improving sexual dysfunction. It has been reported that these agents are very useful in reducing the sexual dysfunction due to SSRIs, resulting in increase in libido, lubrication, orgasm and sexual enjoyment (Nurnberg et al 2008). However, these agents fail to correct sexual problems in a number of patients and search is on to identify other remedies. Contrary to general belief, the sexual adverse effects will not subside on stopping the drugs (SSRI and SNRI) and may persist for quite some time after stopping the drugs (Bahrick, 2008). Looking for other causes of sexual dysfunction is also important in these patients.

\section{Drowsiness}

Drowsiness is commonly seen in patient who are on antidepressants (Hu et al 2004). It is more common with TCAs and mirtazapine than with SSRIs and SNRIs. Proper evaluation of patients for sleep patterns and counseling will help to overcome drowsiness. Taking the entire dose at night or dividing the dose in such a way that the one third is taken in morning and two third at bed time or use of a slower release preparation may also help to overcome drowsiness. If no improvement, psychostimulants, modafinil, may be considered.

\section{Insomnia}

About 12-22\% of patients complained of insomnia and most of them (about 64\%) experienced it by two weeks of treatment (Wouters et al 2014, Hu et al 2004). Patients on SSRIs had higher rates of insomnia than patients treated with mirtazapine, trazodone, and nefazodone (Papakostas, 2007). Proper evaluation of patients with insomnia for primary sleep disorders is very much important before changing the medication or treating the insomnia. The treatment for insomnia includes patient education on behavioural techniques and sleep hygiene measures and adding adjunctive medications including sparing use of benzodiazepines. Avoiding beverages that stimulate central nervous system especially in the evening hours also helps to overcome insomnia.

\section{Tremors}

Antidepressants may cause fine tremors of the extremities. Compared to placebo, the rates of tremor is 2-5 times more with antidepressants. The tremors are more with SSRIs and venlafaxine than with nefazodone and mirtazapine (Nelson,1997). However, other causes of tremors, such as anxiety or excessive caffeine intake must be ruled out before considering it as a side effect of antidepressants. In the absence of other causes, decreasing caffeine intake and use of $\beta$-blockers can be helpful in the treatment of tremors.

\footnotetext{
Apathy

Described as indifference to surrounding is a troublesome adverse effect. It may include loss of motivation or dullness, often develops slowly, and probably due to an antidopaminergic effect of serotonergic medications (Hoehn-Saric et al, 1990). Treatment of apathy includes reduction in dose, changing to a different drug or class or adding a dopaminergic drug. If not controlled by these measures, one can think of using methylphenidate, dextroamphetamine, modafinil or pergolide for control of apathy.

Hyperprolactinemia

Antidepressants have variable effects on prolactin ranging from mild increase to no effect (Coker and Taylor, 2010). Fluoxetine is known to only modestly elevate prolactin levels (Papakostas et al 2006). Bupropion and mirtazapine, do not have any effect on prolactin secretion. Tricyclic antidepressants are known to induce mild increase in prolactin secretion (Torre and Falorni, 2007). In men, symptoms of erectile dysfunction and decreased libido
} 
(Kennedy and Rizvi, 2009) and in women, infertility, decreased libido, galactorrhoea, decrease in bone mineral density, menstrual irregularities are seen. In confirmed cases of hyperprolactinemia, a change to mirtazapine may be a good alternative (Carvalho et al, 2016). In selected cases, a switch to another SSRI may help to control hyperprolactinemia (Mondal et al 2013).

\section{The discontinuation syndrome}

Abrupt discontinuation of antidepressants treatment may precipitate a discontinuation syndrome. The discontinuation syndrome may be seen in hours or days after stopping the treatment. The incidence varies from $5 \%$ to $86 \%$ and develops within a week after down titrating the dose or stopping SSRI or SNRI (Mondal et al 2013, Andrade et al, 2010). The symptoms include malaise, myalgia, nausea, dizziness, and headache, and may even include neurologic symptoms such as unsteady gait, dysesthesias such as unusual shock-like sensations, tremulousness, or vertigo (Kinrys et al, 2004, Zajecka et al, 1997). This syndrome is more common with antidepressants with short half-life (Renoir, 2013). Individualization of dose of specific antidepressant is likely to reduce the chances of discontinuation syndrome. Putting the patient on SSRI with a longer half-life (eg, fluoxetine) while discontinuing the treatment might be helpful in reducing the discontinuation symptoms.

\section{Conclusion:-}

Antidepressants are known to cause a number of side effects which often results in poor patient compliance. More often, the clinicians miscalculate the prevalence of adverse effects and do not act proactively to reduce them out of a mistaken concern that their impact will be magnified. Hence, the adverse effects due to antidepressants are a real challenge to clinicians. Proper patient education prior to and throughout the treatment with antidepressants will definitely help to reduce the incidence of adverse effects.. Additionally, one should be able to differentiate the side effects from the disease related symptoms and other comorbid conditions; and recognise them early and manage them appropriately. Finally, the complete strategy includes dose titration, proper timing of doses and willingness to consider changing to other agents. Comprehensive and inventive method of treating adverse effects is a vital factor in attaining depressive remission and improving patient wellbeing and quality of life.

\section{References:-}

1. Andrade, C., Sandarsh, S., Chethan, K. B., \& Nagesh, K. S. (2010). Serotonin reuptake inhibitor antidepressants and abnormal bleeding: a review for clinicians and a reconsideration of mechanisms. The Journal of clinical psychiatry, 71(12), 1565-1575.

2. Bahrick, A. S. (2008). Persistence of sexual dysfunction side effects after discontinuation of antidepressant medications: Emerging evidence. The Open Psychology Journal, 1(1).

3. Carvalho, A. F., Sharma, M. S., Brunoni, A. R., Vieta, E., \& Fava, G. A. (2016). The safety, tolerability and risks associated with the use of newer generation antidepressant drugs: a critical review of the literature. Psychotherapy and psychosomatics, 85(5), 270-288.

4. Clayton, A. H., Pradko, J. F., Croft, H. A., Montano, C. B., Leadbetter, R. A., Bolden-Watson, C., Metz, A. (2002). Prevalence of sexual dysfunction among newer antidepressants. The Journal of clinical psychiatry.

5. Coker, F., \& Taylor, D. (2010). Antidepressant-induced hyperprolactinaemia. CNS drugs, 24(7), 563574.

6. Demyttenaere, K., Enzlin, P., Dewe, W., Boulanger, B., De Bie, J., De Troyer, W., \& Mesters, P. (2001). Compliance with antidepressants in a primary care setting, 1: beyond lack of efficacy and adverse events. Journal of Clinical Psychiatry, 62, 30-33.

7. Demyttenaere, K., \& Jaspers, L. (2008). Bupropion and SSRI-induced side effects. Journal of Psychopharmacology, 22(7), 792-804.

8. Hoehn-Saric, R., Lipsey, J. R., \& McLeod, D. R. (1990). Apathy and indifference in patients on fluvoxamine and fluoxetine. Journal of clinical psychopharmacology.

9. Hu, X. H., Bull, S. A., Hunkeler, E. M., Ming, E., Lee, J. Y., Fireman, B., \& Markson, L. E. (2004). Incidence and duration of side effects and those rated as bothersome with selective serotonin reuptake inhibitor treatment for depression: patient report versus physician estimate. The Journal of clinical psychiatry, 65(7), 959-965.

10. Kelly, K., Posternak, M., \& Jonathan, E. A. (2008). Toward achieving optimal response: understanding and managing antidepressant side effects. Dialogues in clinical neuroscience, 10(4), 409. 
11. Kennedy, S. H., \& Rizvi, S. (2009). Sexual dysfunction, depression, and the impact of antidepressants. Journal of clinical psychopharmacology, 29(2), 157-164.

12. Kinrys G., Simon NM., Farach FJ., Pollack MH. Management of antidepressant- induced side effects. In: Alpert JE, Fava M, eds. . Handbook of Chronic Depression. New York, NY: Marcel Dekker, Inc. 2004:411-446.

13. La Torre, D., \& Falorni, A. (2007). Pharmacological causes of hyperprolactinemia. Therapeutics and Clinical Risk Management, 3(5), 929.

14. Marcy, T. R., \& Britton, M. L. (2005). Antidepressant-induced sweating. Annals of Pharmacotherapy, 39(4), 748-752.

15. Matreja, P. S., Badyal, D. K., Deswal, R. S., \& Sharma, A. (2012). Efficacy and safety of add on lowdose mirtazapine in depression. Indian journal of pharmacology, 44(2), 173.

16. Mondal, S., Saha, I., Das, S., Ganguly, A., Das, D., \& Tripathi, S. K. (2013). A new logical insight and putative mechanism behind fluoxetine-induced amenorrhea, hyperprolactinemia and galactorrhea in a case series. Therapeutic advances in psychopharmacology, 3(6), 322-334.

17. Montejo-Gonzalez, A. L., Llorca, G., Izquierdo, J. A., Ledesma, A., Bouso o, M., Calcedo, A., De la Gandara, J. (1997). SSRI-induced sexual dysfunction: fluoxetine, paroxetine, sertraline, and fluvoxamine in a prospective, multicenter, and descriptive clinical study of 344 patients. Journal of Sex and Marital Therapy, 23, 176-194.

18. Montejo, A. L., Llorca, G., Izquierdo, J. A., \& Rico-Villademoros, F. (2001). Incidence or sexual dysfunction associated with antidepressant agents: A prospective multicenter study of 1022 outpatients. The Journal of clinical psychiatry.

19. Nelson, J. C. (1997). Safety and tolerability of the new antidepressants. The Journal of clinical psychiatry, 58, 26-31.

20. Nurnberg, H. G., Hensley, P. L., Heiman, J. R., Croft, H. A., Debattista, C., \& Paine, S. (2008). Sildenafil treatment of women with antidepressant-associated sexual dysfunction: a randomized controlled trial. Jama, 300(4), 395-404.

21. Papakostas, G. I., Miller, K. K., Petersen, T., Sklarsky, K. G., Hilliker, S. E., Klibanski, A., \& Fava, M. (2006). Serum prolactin levels among outpatients with major depressive disorder during the acute phase of treatment with fluoxetine. The Journal of clinical psychiatry, 67(6), 952-957.

22. Papakostas, G. I. (2007). Limitations of contemporary antidepressants: tolerability. The Journal of clinical psychiatry, 68, 11-17.

23. Renoir, T. (2013). Selective serotonin reuptake inhibitor antidepressant treatment discontinuation syndrome: a review of the clinical evidence and the possible mechanisms involved. Frontiers in pharmacology, 4, 45.

24. Rothschild, A. J. (1995). Selective serotonin reuptake inhibitor-induced sexual dysfunction: efficacy of a drug holiday. The American journal of psychiatry.

25. Sinclair, L. I., Christmas, D. M., Hood, S. D., Potokar, J. P., Robertson, A., Isaac, A., ... \& Davies, S. J. (2009). Antidepressant-induced jitteriness/anxiety syndrome: systematic review. The British Journal of Psychiatry, 194(6), 483-490.

26. Uguz, F. (2013). Low-dose mirtazapine added to selective serotonin reuptake inhibitors in pregnant women with major depression or panic disorder including symptoms of severe nausea, insomnia and decreased appetite: three cases. The Journal of Maternal-Fetal \& Neonatal Medicine, 26(11), 1066-1068.

27. WHO. Depression In: Organization WH, (ed), 2017.

28. Wang, S. M., Han, C., Bahk, W. M., Lee, S. J., Patkar, A. A., Masand, P. S., \& Pae, C. U. (2018). Addressing the side effects of contemporary antidepressant drugs: a comprehensive review. Chonnam medical journal, 54(2), 101-112.

29. Wouters, H., Van Dijk, L., Van Geffen, E. C. G., Gardarsdottir, H., Stiggelbout, A. M., \& Bouvy, M. L. (2014). Primary-care patients' trade-off preferences with regard to antidepressants. Psychological medicine, 44(11), 2301-2308.

30. Wouters, H., Bouvy, M. L., Van Geffen, E. C., Gardarsdottir, H., Stiggelbout, A. M., \& Van Dijk, L. (2014). Antidepressants in primary care: patients' experiences, perceptions, self-efficacy beliefs, and nonadherence. Patient preference and adherence, 8, 179.

31. Zajecka, J., Tracy, K. A., \& Mitchell, S. (1997). Discontinuation symptoms after treatment with serotonin reuptake inhibitors: a literature review. The Journal of clinical psychiatry, 58:291-297.

32. Zajecka, J. (2001). Strategies for the treatment of antidepressant-related sexual dysfunction. The Journal of clinical psychiatry, 62, 35-43. 